\title{
Prevention of temporary emergency medical center closure through isolation zone and screening triage in COVID-19 outbreak
}

\author{
Sin-Youl Park ${ }^{1}$, Kyung Woo Lee ${ }^{2, *}$, Suk Hee Lee ${ }^{2}$, Jong Kun Kim³, \\ Jae Wan $\mathrm{Cho}^{3}$, Jae Cheon Jeon ${ }^{4}$, Jae Chul Cho ${ }^{5}$
}

\author{
${ }^{1}$ Department of Emergency Medicine, \\ Yeungnam University College of \\ Medicine, 42415 Daegu, \\ Republic of Korea \\ ${ }^{2}$ Department of Emergency Medicine, \\ Catholic University of Daegu School of \\ Medicine, 42472 Daegu, \\ Republic of Korea \\ ${ }^{3}$ Department of Emergency Medicine, \\ School of Medicine, Kyungpook National \\ University, 41944 Daegu, \\ Republic of Korea \\ ${ }^{4}$ Department of Emergency Medicine, \\ Keimyung University School of Medicine, \\ 42601 Daegu, Republic of Korea \\ ${ }^{5}$ Department of Emergency Medicine, \\ Daegu Fatima Hospital, 41199 Daegu, \\ Republic of Korea \\ *Correspondence \\ turtle072@hanmail.net \\ (Kyung Woo Lee)
}

\begin{abstract}
Background: In Korea, temporary emergency medical center (EMC) closures are used to prevent nosocomial transmission of COVID-19, but they can harm the local emergency medical system. This study aimed to evaluate the occurrence of temporary EMC closures and the adequacy of isolation zone and screening triage during the COVID-19 outbreak.

Methods: This retrospective study was conducted in Daegu, Korea from 18 February 2020 to 30 April 2020, in patients who caused temporary EMC closures. The distribution of EMC closures according to time and isolation zone installation, screening triage by four criteria (epidemiologic link, fever, respiratory symptoms, unknown pneumonia), reasons for RT-PCR SARS-CoV-2 tests, and causes of EMC closures was analysed.

Results: There were a total of 26 temporary EMCs closures including eight multi-center closures. Temporary EMCs closures occurred frequently in the early stages of outbreak. Temporary EMC closure was made without RT-PCR SARS-CoV-2 confirmation. Before and after isolation zone installation, there were 24 and two temporary EMC closures, respectively. Respiratory symptoms were the most common basis for identification at screening triage centers, and fevers were the symptoms most commonly recognized by clinicians. Most (88.5\%) patients causing closures met one or more of the four criteria at EMC admission. The most common cause of temporary EMC closures was patient complaint of prominent symptoms suggestive of another disease regardless of presence of screening criteria (18 cases, $69.2 \%$ ).

Conclusions: Isolation zone installation and strictly applying the four criteria individually in screening triage is useful in reducing temporary EMC closure.
\end{abstract}

\section{Keywords}

Coronavirus disease 2019 (COVID-19); Emergency medicine; Triage; Infection control; Isolation; Severe acute respiratory syndrome coronavirus 2 (SARS-CoV-2); South Korea

\section{Introduction}

With vaccination, confirmed cases of the severe acute respiratory syndrome coronavirus 2 (SARS-CoV-2) in 2019 (COVID$19)$ is rapidly decreasing and stabilizing in countries such as the United States and Israel. However, in countries where vaccination is still scarce, large-scale COVID-19 outbreaks are occurring persistently and recurrently.

As of April 2021, South Korea is undergoing its fourth COVID-19 outbreak, with 500 to 700 new COVID confirmed cases per day. The first case of COVID-19 in South Korea was a patient who returned home from Wuhan, China on 19 January 2020 [1]. The first domestic outbreak occurred in Daegu on 18 February 2020. Daegu, with a population of 2.48 million, is a city with six emergency medical centers (EMCs) (Two Level I EMCs and four Level II EMCs) providing emergency medical services to approximately 200,000 patients [2]. During the first outbreak, (18 February-30 April 2020) in South Korea, 10,774 confirmed cases and 248 deaths were reported nationwide, and a total of $6852(63.6 \%)$ and $172(172 / 248)$ deaths occurred in Daegu [3].

Prior to the first COVID-19 outbreak the Korea Centers for Disease Control and Prevention (KCDC), prepared early recognition and pre-emptive isolation of COVID-19 in EMCs by installing screening triage clinics (STCs) and negative pressure isolation rooms in EMCs. In addition, KCDC established and distributed screening criteria for early recognition of suspected COVID-19 patients. Suspected COVID-19 patients were defined as patients who developed symptoms within 14 days of visiting overseas or contacting a confirmed patient, or who developed symptoms after epidemiological contact with a domestic COVID-19 outbreak. Symptoms of COVID-19 
patients included respiratory symptoms such as body temperature $\geq 37.5^{\circ} \mathrm{C}$, cough, shortness of breath, sore throat, and unknown pneumonia [4, 5].

Inappropriate screening triage may lead to nosocomial transmission through EMC exposure to COVID-19. In 2015, South Korea experienced 186 infections and 38 deaths due to nosocomial transmission of Middle East respiratory syndrome coronavirus (MERS-CoV), from patients who were not recognized early through screening tests [6]. At the time of the MERS-CoV outbreak, hospitals closed EMCs to stop uncontrolled nosocomial transmission of MERS-CoV from EMCs, and these measures helped prevent the nosocomial transmission of MERS-CoV [7]. Based on these experiences, hospitals in South Korea are performing temporary EMC closures to prevent the spread of SARS-CoV-2 to patients and healthcare workers in situations where an EMC is exposed to COVID-19. However, because EMC closure causes sudden loss of essential emergency resources, it can cause serious disruption to local emergency medical systems [8]. Therefore, efforts are needed to make judicious decisions about EMC closures.

To help prevent inappropriate temporary EMC closures in response to nosocomial transmission of COVID-19, this study investigated the occurrence and distribution of temporary EMC closures and evaluated the adequacy of screening triage and isolation during COVID-19 outbreak.

\section{Methods}

\subsection{Study population and data collection}

This retrospective study enrolled patients who caused temporary EMC closures in Daegu, South Korea from 18 February 2020 , to 30 April 2020. EMCs that were partially or unofficially closed were excluded. The attending physician at each participating EMC collected the clinical data of the patients from the medical records including radiology and laboratory results. For anonymity, personally identifiable numbers were removed from the collected data, and a new number was randomly assigned to each hospital-specific number. The accuracy and anonymity of collected data were re-evaluated by two researchers. Information on the number of confirmed COVID-19 patients per day was obtained from the Daegu City Health Department and KCDC reports.

\subsection{Ethics statement}

The Institutional Review Board (IRB) at Daegu Catholic University Hospital reviewed and approved the study protocol (IRB No. CR-20-146). After that, the IRB at each of the participating hospitals approved the study.

\subsection{Definition of temporary EMC closure, Multi-center closure, STC and Isolation zone}

Temporary EMC closure was defined as the complete cessation of patient entry and exit from an EMC until quarantine and decontamination were complete. Temporary EMC closure period was the period from the official EMC closure to the resumption of patient visits to the EMC. Multi-center closure was defined as closures of three or more EMCs over the same period.

An STC was a temporary clinic installed at the entrance of an EMC with a negative pressure facility. The main roles of STCs were screening triage, implementation of portable chest radiography (CXR), and specimen collection for reverse transcription-polymerase chain reaction (RT-PCR) for SARSCoV-2.

An isolation zone was an independent quarantine space installed inside or outside an EMC with separate entrances and exits from the EMC. Until confirmation of RT-PCR SARSCoV-2 results, active monitoring, radiologic examination including CXR, laboratory examination and medical care including oxygen therapy could be administered in the isolation zone. There were no negative pressure facilities, but inter-bed distance of $2 \mathrm{~m}$ was maintained, and patients and health care workers (HCWs) wore masks of N94 or higher.

\subsection{Outcome measurements}

\subsubsection{Appropriateness of screening triage}

Screenings were performed separately at STCs and at EMCs, classifying cases into four categories: epidemiologic link, fever, respiratory symptoms, and unknown pneumonia in CXR. Epidemiologic link was positive if an overseas visit, contact with a confirmed COVID-19 patient, or epidemiologic association with a domestic mass occurrence with COVID-19 was identified. If body temperature (BT), measured by noncontact method in the STC or EMC, was $37.5^{\circ} \mathrm{C}$ or higher, it was defined as fever. Records of cough, sputum, sore throat, and dyspnea in the STC or EMC were defined as positive respiratory symptoms. When a pulmonary abnormality suggesting viral pneumonia was observed on the CXR, it was defined as unknown pneumonia in CXR. Chest computed tomography (CT) was used as the diagnostic reference for unknown pneumonia in CXR. If chest CT was not performed within 24 hours after CXR, two thoracic radiologists reviewed the CXRs, independently. In case of disparity a final decision was made, through consensus, by the two experts. In addition, we investigated whether the clinician perceived unknown pneumonia in CXR in an STC or in an EMC. Whether or not the clinician recognized the unknown pneumonia in CXR was determined based on the clinician's STC or EMC record.

\subsubsection{Reasons for RT-PCR SARS-CoV-2 test}

Confirmation of COVID-19 was made by positive RT-PCR SARS-CoV-2 results in respiratory specimens of nasal swabs or sputum (RT PCR kit [KogeneBiotech, Seoul, South Korea] and Allplex 2019-nCoV Assay [Seegene, Seoul, South Korea]). The reasons why the RT-PCR SARS-CoV-2 test was performed in STC or EMC were investigated and classified based on the clinician's records, and the RT-PCR SARS-CoV-2 test time and result notification time were investigated.

\subsubsection{Causes of temporary EMC closure}

The cause of the decision of EMC closure was investigated by exploring the records of each hospital's infection control team. Three emergency medical specialists categorized the results into categories, independently. If the classification results were not consistent, it was decided by agreement of 


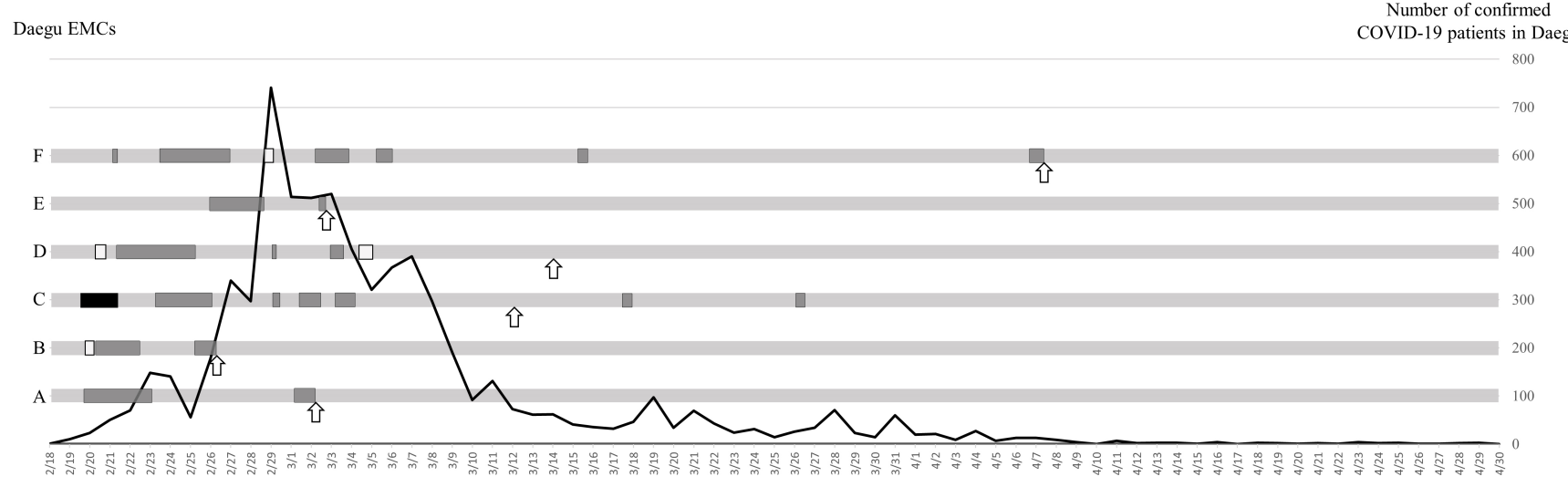

F I GURE 1. The distribution and duration of the temporary emergency medical center (EMC) closure in Daegu according to the number of confirmed coronavirus disease 2019 (COVID-19) patients per day in Daegu. A through $F$ represent the six EMCs in Daegu. The black line represents the number of confirmed COVID-19 patients per day in Daegu. The squares on the gray bands of each EMC indicate the occurrence of a temporary EMC closure and the area of the squares represents the duration. Of these, dark gray squares represent decision of temporary EMC closure after confirmation of reverse transcription-polymerase chain reaction for severe acute respiratory syndrome coronavirus 2 (RT-PCR for SARS-CoV-2). Black and white squares represent the temporary EMC closures were made without confirmation of RT-PCR SARS-CoV-2 test. Black square means that the result of the RT-PCR SARS-CoV-2 test was postive, and the white squares mean that the RT-PCR SARSCoV-2 test results were negative. The arrows indicate the dates the isolation zones were installed in each EMC.

three specialists.

\subsection{Statistical analysis}

In the case of continuous variables, the Shapiro-Wilk test was used to test for the normality of the data. Non-normally distributed data were expressed as the median (interquartile range), and tested with the Mann-Whitney U test. Categorical variables were expressed as frequency (\%), and statistical significance was confirmed by the Chi-squared test or Fisher's exact test. Results were considered statistically significant when the $P$-value was less than 0.05 . Statistical analyses were performed using SPSS version 14.0 (SPSS Inc., Chicago, IL, USA).

\section{Results}

There was a total of 26 temporary EMC closures in Daegu between 18 February and 30 April. The first temporary EMC closure occurred when the first COVID-19 patient was confirmed in Daegu. The total period of this temporary closure was 16.4 days. There was a total of eight multi-center closures lasting for 14.2 days. Each EMC was temporarily closed for five days (range, two-six days). The average age of 26 patients who caused temporary EMCs closures was 62.4 years (range, 57-68 years) and 13 (50\%) males. Nine patients (34.6\%) died.

Fig. 1 shows the distribution and duration of temporary EMC closures in Daegu, and the number of COVID-19 cases. In the initial 17 days of the outbreak, 22 cases $(84.6 \%)$ of temporary EMC closure and eight cases (100\%) of multi-center closure occurred. Of the 26 temporary EMC closures, five $(19.2 \%)$ were made without confirmation via RT-PCR SARSCoV-2 test, and four of these cases $(80 \%)$ were later confirmed negative by RT-PCR SARS-CoV-2 results.
An isolation zone was installed after an average of 3.7 temporary EMC closures. The first isolation zone was installed on 26 February. There were 24 and two temporary EMCs closures, respectively, before and after the installation of an isolation zone. The occurrence of temporary EMC closures in EMCs which installed isolation zones was significantly reduced $(P<0.01)$.

Screening triage results for suspected COVID-19 patients are shown in Table 1. There was no significant difference between STC and EMC in identifying three screening criteria (fever, $P=0.53$; respiratory symptoms, $P=0.77$; pulmonary abnormality in CXR, $P=0.34)$. Of the 26 cases, five $(19.2 \%)$ were confirmed to have epidemiologic links, but none of them were recognized at an STC, while five cases of epidemiologic links were identified after positive RT-PCR SARS-CoV-2 results at the EMCs $(P=0.03)$. All of these were directly or indirectly associated with a certain religious cluster. Since March 2nd, no epidemiological relationship has been identified for a patient's COVID-19. A total of 20 patients (76.9\%) were identified with fever, 11 (42.3\% of total) were identified in an STC. The median BT measured at STC was 37.1 (range, 36.6-37.8). A total of 24 patients $(92.3 \%)$ had respiratory symptoms. Respiratory symptoms of 17 patients (65.4\%) were identified at STCs. The other nine patients were as follows; two had flank pain, two had diarrhea, two had hip pain with fractures, one had neck pain, one had a loss of consciousness, and one had hyperglycemia. Of these, in seven patients, respiratory symptoms were identified during treatment in the EMC. CXR was performed in the STCs and EMCs on five and 21 patients, respectively. Of the 26 patients, 14 (53.8\%) had chest CTs. In CXR, pulmonary abnormalities suggesting viral pneumonia were found in a total of 18 patients $(69.2 \%)$, three out of five patients $(60 \%)$ at an STC and 15 out of 21 patients $(71.4 \%)$ at an EMC. Clinicians were able to detect 
TA B L E 1. Results of screening and triage according to screening criteria.

\begin{tabular}{lcccc} 
& Total (n, \%) & Screening triage clinic (n, \%) & Emergency medical center (n, \%) & $P$ \\
Epidemiologic links & $5(19.2)$ & $0(0 / 26,0)$ & $5(5 / 26,19.2)$ & 0.03 \\
Fever $^{a}$ & $20(76.9)$ & $11(11 / 26,42.3)$ & $9(9 / 15,60.0)$ & 0.53 \\
Respiratory symptoms & $24(92.3)$ & $17(17 / 26,65.4)$ & $7(7 / 9,77.7)$ & 0.77 \\
Pulmonary abnormality in CXR & $4(15.4)$ & $0(0 / 5,0)$ & $4(4 / 21,19.0)$ & 0.34 \\
\hline
\end{tabular}

$C X R$, chest radiography.

${ }^{a}$ Fever, body temperature $>37.5^{\circ} \mathrm{C}$.

four cases $(22.2 \%)$ of $18 \mathrm{CXRs}$ with pulmonary abnormalities (zero out of three at STCs and four out of 15 at EMCs (26.7\%). For pulmonary abnormalities, ground-glass opacity (GGO) and consolidation (multifocal or patchy) were observed in 13 $(72.2 \%)$ and five $(27.8 \%)$ patients, respectively. Bilateral lesions were observed in $11(61.1 \%)$ of the 18 patients with pulmonary abnormalities.

Table 2 shows the distribution of the number of screening criteria identified in patients who caused temporary EMCs closures. Of the 26 patients, $23(88.5 \%)$ met at least one of the four screening criteria in the STC, however, none met all four screening criteria. Three patients $(11.5 \%)$ did not meet any of the four screening criterion. Six patients (23.1\%) met three screening criteria, 11 patients $(42.3 \%)$ met two screening criteria, while six patients $(23.1 \%)$ met only one criterion. All 26 patients met at least one of the four screening criteria when the additionally identified cases in the EMCs were included. Among these there were three patients (11.5\%) meeting all four criteria, 12 patients $(46.2 \%)$ meeting three criteria, six patients $(23.1 \%)$ meeting two criteria, and five patients $(19.2 \%)$ meeting one criterion.

The RT-PCR SARS-CoV-2 test took an average of 366 minutes to perform after arrival at the STC. After the RT-PCR SARS-CoV-2 test, it took an average of 14.7 hours to check the results (external laboratory, 20.2 hours; internal laboratory, 7.9 hours). Nine patients (34.6\%) underwent RT-PCR testing in STCs and 17 patients (65.4\%) underwent RT-PCR testing in EMCs. Table 3 shows the reasons for RT-PCR SARS-CoV-2 tests at the STCs and EMCs. In both the STC and EMC groups, fever was the most common reason for RT-PCR SARS-CoV-2 testing $(P=0.003)$.

The causes of temporary EMC closures are shown in Table 4. The most common cause of temporary EMC closures was when a patient complained of prominent symptoms suggestive of another disease (18 cases $(69.2 \%), P=0.03)$. Six cases $(23.1 \%)$ of temporary EMC closure were due to lack of space to treat an exacerbated condition in suspected COVID-19 patients. The lack of an isolation room and falsenegative COVID-19 tests conducted by other hospitals were investigated as one cause of temporary EMC closure each.

\section{Discussion}

Crowding, a chronic problem in the emergency department (ED), is one of the causes of ED closures [9]. In the COVID19 outbreak, hospitals in South Korea used temporary EMC closure to prevent the spread of the virus, as in the MERS ex- perience in 2015 [7], and temporary EMC closure is considered to have effectively controlled the nosocomial transmission of COVID-19 [10, 11].

However, EMC closures can have a poor outcome for patients with time-sensitive illnesses, such as acute myocardial infarction [12]. During this COVID-19 outbreak, there is a report that the duration of ED stay in emergency patients was prolonged and the number of severely ill patients increased in EMC closures [10]. In addition, EMC closure can lead to reduced regional emergency medical resources and the resulting crowding and overburden of nearby EMCs [9]. This can lead to multi-center closure in the region, making the situation even worse regionally. In the COVID-19 outbreak, temporary EMC closures were significantly associated with increased community hospital mortality and ICU hospitalization rates, and extended ED stays [8]. Therefore, the decision to temporarily EMC close must be made carefully.

Accurate screening triage can be the key factor in reducing EMC closure. In the early stages of a highly contagious virus outbreak, identifying the patient's epidemiologic links is one of the most important roles of the STCs. In KDCD guidelines, epidemiologic link is a must-have screening criterion for recognizing suspected COVID-19 patients. However, this study showed epidemiologic links were difficult to identify in STC. When epidemiologic links are related to privacy issues, identification becomes difficult. The spread of COVID-19 in Daegu was closely related to heretical religious groups that are reluctant to reveal their affiliation [13]. Even if there are no epidemiological links, COVID-19 should not be ruled out.

Body temperature $\geq 37.8{ }^{\circ} \mathrm{C}(56.4 \%-63.3 \%)$ and cough $(52.7 \%-59.2 \%)$ were the most frequently presenting symptoms in hospitalized COVID-19 patients [14, 15]. Consistent with these results, this study showed that in patients that caused temporary EMC closures, respiratory symptoms (65.4\%) and fever (42.3\%) were the most common symptoms found in STC. Respiratory symptoms are an important clue to COVID-19 because it primarily affects the respiratory system [16, 17]. In agreement with these findings, in the present study, $92.3 \%$ of the patients presented with respiratory symptoms. However, clinicians at STC did not consider respiratory symptoms an important reason for RT-PCR SARS-CoV-2 tests and focused on patients' main symptoms. This is supported by the fact that respiratory symptoms account for only $11.5 \%$ of reasons for a RT-PCR SARS-CoV-2 test and 34.6\% patients causing temporary EMC closures had main symptoms unrelated to respiratory symptoms. Evaluating patients based on the main symptoms is common in EDs, but in the COVID-19 outbreak, 
TA B L E 2. Number of screening criteria identified in patients who caused temporary emergency medical center closures.

\begin{tabular}{lcc} 
Number of screening criteria & Total $(\mathrm{n}, \%)$ & Screening triage clinic (n, \%) \\
4 & $3(11.5)$ & $0(0)$ \\
3 & $12(46.2)$ & $6(23.1)$ \\
2 & $6(23.1)$ & $11(42.3)$ \\
1 & $5(19.2)$ & $6(23.1)$ \\
0 & $0(0)$ & $3(11.5)$ \\
\hline
\end{tabular}

TA B L E 3. Reasons for RT-PCR SARS-CoV-2 tests of patients causing the temporary emergency medical center closures.

\begin{tabular}{|c|c|c|c|c|}
\hline Reasons for RT-PCR tests & \multicolumn{3}{|c|}{ Total $(n=26)$ Screening triage clinic $(n=9)$ Emergency medical center $(n=17)$} & $P$ \\
\hline Fever $^{a}$ & $17(65.4)$ & $4(44.5)$ & $13(76.5)$ & \multirow{4}{*}{0.003} \\
\hline Respiratory symptoms & $3(11.5)$ & $3(33.3)$ & $0(0)$ & \\
\hline Screening purpose & $2(7.7)$ & $2(22.2)$ & $0(0)$ & \\
\hline Pulmonary abnormality in CXR & $4(15.4)$ & $0(0)$ & $4(23.5)$ & \\
\hline
\end{tabular}

$R T-P C R$, real-time reverse transcriptase-polymerase chain reaction; SARS-CoV-2, severe acute respiratory syndrome coronavirus 2; CXR, chest radiography.

${ }^{a}$ Fever, body temperature $>37.5^{\circ} \mathrm{C}$.

TA B L E 4. Causes of COVID-19 patients entering the EMCs without RT-PCR SARS-CoV-2 result.

$\begin{array}{lc}\text { Causes of COVID-19 patients entering the EMCs } & \text { Total }(\mathrm{n}=26) \\ \text { No suspicion and mistaken for other diseases } & 18 \\ \text { Lack of space to treat an exacerbated condition } & 6 \\ \text { Lack of isolation rooms } & 1 \\ \text { Negative COVID-19 test conducted by another hospital } & 1 \\ \text { COVID-19, coronavirus disease 2019; RT-PCR, real-time reverse transcriptase- } \\ \text { polymerase chain reaction; SARS-CoV-2, severe acute respiratory syndrome } \\ \text { coronavirus 2; EMCs, emergency medical centers. }\end{array}$

the clinician should carefully monitor the accompanying respiratory symptoms separately from the patient's main symptoms. The present study showed that fever was the most common reason for performing RT-PCR SARS-CoV-2 tests for COVID19 confirmation. Fever was considered by clinicians the most accessible and reliable criterion among the screening criteria, because body temperature can be easily measured and the result is an objective value. In an early study of COVID-19 pandemic, there are reports that a fever was found in $94 \%$ of patients at hospitalization $[16,17]$. However, in this study, a total of $23.1 \%$ of the patients had no fever, despite a positive RT-PCR result. Similarly, in the 2019 Case Surveillance in the United States, only $43.1 \%$ of COVID-19-positive patients had a fever of $38{ }^{\circ} \mathrm{C}$ or higher at admission [18]. These results suggest that the absence of fever does not rule out COVID-19.

To confirm COVID-19 pneumonia, the KCDC recommended the use of CXR at the STCs. CXR is the basic imaging modality for identifying pneumonia. Because CXR equipment is portable, it is unlikely that the virus will spread into hospitals due to testing [19]. Therefore, CXR may be suitable as an initial imaging modality for COVID-19 pneumonia in STCs [20]. However, a consideration for CXR is its low sensitivity to COVID-19 pneumonia. The sensitivity of CXR for COVID-19 pneumonia was reported to be $69 \%$ to $75 \%$, and because of the low sensitivity, several studies have even suggested that CXR should not be the primary imaging modality for COVID-19 [21, 22]. Chest CT is a useful diagnostic modality for COVID-19 pneumonia $[23,24]$. In a study of patients hospitalized with COVID-19, chest CT scans improved the identification of pneumonia from $30.9 \%-34.7 \%$ to $65.3-69.1 \%$ [14, 15]. GGO lesions, the most common finding of CXR in COVID-19, are not clearly identified, making it difficult for clinicians to detect them in CXR. In this study, clinicians identified only $22.2 \%$ of CXR with COVID-19 pneumonia. Therefore, active application of chest CT can be useful for early identification of COVID-19 pneumonia. There were differences in chest CT applications between Daegu EMCs. This is because safety protocols had not been established to prevent the spread of viruses and CT room contamination during patient transport.

In the surge of suspected COVID-19 patients, negative pressure isolation rooms were quickly saturated. Lack of treatment, testing, and observation space for suspected COVID19 patients with low or moderate risk can lead to increased possibility of exposure of the EMC to COVID-19, which in turn led to EMC closures. Isolation zones have played an 
important role in solving the problem of lack of treatment, testing, and observation space. As confirmed in this study, occurrence of EMC closures after the installation of isolation zone was significantly reduced. Since there was no negative pressure facility in the isolation zones of Daegu EMCs, there may be a risk of transmission of SARS-CoV-2 within the zone. However, there have as yet been no reports of COVID19 nosocomial transmission through the isolation zones. The isolation zones were operated with an inter-bed distance of 2 m, and patients (N94 mask) and HCW (N94 mask and N95 mask) wore masks in accordance with the KCDC guidelines. Several studies reported that social distancing within hospitals and universal masking of patients and health care workers are useful for the prevention of nosocomial transmission of COVID-19 [25, 26]. Since 11 March 2020, the KCDC has encouraged EMCs across the country to install isolation zones with social distancing between beds, and that in hospitals all people are required to wear masks of N94 grade or higher.

There were several limitations to our study. First, due to the retrospective nature of the study, there is a possibility of research bias. Second, only 26 patients from our cohort were included in this study. The small sample size might limit the generality of the results. To reduce selection bias, this study was performed by anonymizing all data, and the collected data were evaluated by experts who did not take part in the collection of the initial data. Third, this study has a territorial limit. Since this study describes the experience of EMCs in a city, the results may not be generalizable. The study is nonetheless meaningful as it has detailed data related to the official temporary closure of the EMCs in Daegu, which was the epicenter for COVID-19 in South Korea.

\section{Conclusions}

To reduce exposure of EMC to SARS-CoV-2, clinicians in the STC should apply four screening criteria strictly individually, raising suspicions of COVID-19. Isolation zones with interbed distance of $2 \mathrm{~m}$ and wearing masks in the isolation zone were useful to reduce the temporary EMC closure through solving the lack of space.

\section{AUTHOR CONTRIBUTIONS}

SYP, KWL, SHL, JKK, JWC, JCJ and JCC contributed to the data collection, analyzing the results and reviewed the final manuscript. SYP provided the initial draft of the manuscript and statistical analysis. KWL contributed to the final draft preparation of the manuscript and article submission as the corresponding author.

\section{ETHICS APPROVAL AND CONSENT TO PARTICIPATE}

The Institutional Review Board (IRB) at Daegu Catholic University Hospital reviewed and approved the study protocol (IRB No. CR-20-146). After that, the IRB at each of the participating hospitals approved the study.

\section{ACKNOWLEDGMENT}

We thank to all the peer reviewers for their opinions and suggestions.

\section{FUNDING}

This work was supported by the Research Program of Medicity Daegu Council funded by Daegu Metropolitan City (COVID19_DM17).

\section{CONFLICT OF INTEREST}

The authors declare no conflict of interest.

\section{DATA AVAILABILITY}

The data used to support the findings of this study are available from the corresponding author upon request.

\section{REFERENCES}

[1] Kim JY, Choe PG, Oh Y, Oh KJ, Kim J, Park SJ, et al. The first case of 2019 novel coronavirus pneumonia imported into Korea from Wuhan, China: implication for infection prevention and control measures. Journal of Korean Medical Science. 2020; 35: e61.

[2] Daegu Metropolitan City. Current status of emergency medicalcare center. Available at: https://www.daegu.go.kr/health/index. do?menu_id=00933174 (Accessed date: 10 January 2021)

[3] Korea Centers for Disease Control and Prevention. Updates on COVID19 in Republic of Korea (as of 1 May). 2019. Available at: http: //ncov.mohw.go.kr/en/tcmBoardView.do? (Accessed: 10 January 2021).

[4] Hong KH, Lee SW, Kim TS, Huh HJ, Lee J, Kim SY, et al. Guidelines for Laboratory Diagnosis of Coronavirus Disease 2019 (COVID-19) in Korea. Annals of Laboratory Medicine. 2020; 40: 351-360.

[5] Korean Society of Infectious Diseases; Korean Society of Pediatric Infectious Diseases; Korean Society of Epidemiology; Korean Society for Antimicrobial Therapy; Korean Society for Healthcare-associated Infection Control and Prevention; Korea Centers for Disease Control and Prevention. Report on the Epidemiological Features of Coronavirus Disease 2019 (COVID-19) Outbreak in the Republic of Korea from January 19 to March 2, 2020. Journal of Korean Medical Science. 2020; 35: e112.

[6] Kwon S, Lee E, Kim H, Kim J, Lim Y, Choi G. Middle East respiratory syndrome coronavirus (MERS-COV) outbreak in South Korea: risk management at the Korean Red Cross Seoul Nambu Blood Center: 3BS11-05. Vox Sanguinis. 2015; 109: 18-19.

[7] Kim JY, Song JY, Yoon YK, Choi SH, Song YG, Kim SR, et al. Middle East Respiratory Syndrome Infection Control and Prevention Guideline for Healthcare Facilities. Infection \& Chemotherapy. 2015; 47: 278-302.

[8] Lee DE, Ro YS, Ryoo HW, Moon S. Impact of temporary closures of emergency departments during the COVID-19 outbreak on clinical outcomes for emergency patients in a metropolitan area. The American Journal of Emergency Medicine. 2021; 47: 35-41.

[9] Kim YJ, Choe JY, Kwon KT, Hwang S, Choi G-S, Sohn JH, et al. How to keep patients and staff safe from accidental SARS-CoV-2 exposure in the emergency room: Lessons from South Korea's explosive COVID-19 outbreak. Infection Control \& Hospital Epidemiology. 2021; 42: 18-24.

[10] Lee JH, Hong SW, Hyun M, Park JS, Lee JH, Suh YS, et al. Epidemiological and clinical characteristics of coronavirus disease 2019 in Daegu, South Korea. International Journal of Infectious Diseases. 2020; 98: 462-466.

[11] Shen YC, Hsia RY. Association between Emergency Department Closure 
and Treatment, Access, and Health Outcomes among Patients with Acute Myocardial Infarction. Circulation. 2016; 134: 1595-1597.

[12] Sun BC, Mohanty SA, Weiss R, Tadeo R, Hasbrouck M, Koenig W, et $a l$. Effects of hospital closures and hospital characteristics on emergency department ambulance diversion, Los Angeles County, 1998 to 2004. Annals of Emergency Medicine. 2006; 47: 309-316.

[13] Bostock B. South Korea is testing 200,000 members of a doomsday church linked to more than $60 \%$ of its coronavirus cases. Business Insider. 2020. (25 February 2020)

[14] Hong KS, Lee KH, Chung JH, Shin KC, Choi EY, Jin HJ, et al. Clinical Features and Outcomes of 98 Patients Hospitalized with SARS-CoV2 Infection in Daegu, South Korea: a Brief Descriptive Study. Yonsei Medical Journal. 2020; 61: 431-437.

[15] Jang JG, Hur J, Choi EY, Hong KS, Lee W, Ahn JH. Prognostic Factors for Severe Coronavirus Disease 2019 in Daegu, Korea. Journal of Korean Medical Science. 2020; 35: e209.

[16] Guan WJ, Ni ZY, Hu Y, Liang WH, Ou CQ, He JX, et al. Clinical Characteristics of Coronavirus Disease 2019 in China. New England Journal of Medicine. 2020; 382: 1708-1720.

[17] Huang C, Wang Y, Li X, Ren L, Zhao J, Hu Y, et al. Clinical features of patients infected with 2019 novel coronavirus in Wuhan, China. The Lancet. 2020; 395: 497-506.

[18] Stokes EK, Zambrano LD, Anderson KN, Marder EP, Raz KM, El Burai Felix S, et al. Coronavirus Disease 2019 Case Surveillance United States, January 22-May 30, 2020. Morbidity and Mortality Weekly Report. 2020; 69: 759-765.

[19] Dennie C, Hague C, Lim RS, Manos D, Memauri BF, Nguyen ET, et al. Canadian Society of Thoracic Radiology/Canadian Association of Radiologists Consensus Statement Regarding Chest Imaging in Suspected and Confirmed COVID-19. Canadian Association of Radiologists Journal.
2020; 71: 470-481.

[20] Jacobi A, Chung M, Bernheim A, Eber C. Portable chest X-ray in coronavirus disease-19 (COVID-19): a pictorial review. Clinical Imaging. 2020; 64: 35-42.

[21] Wong HYF, Lam HYS, Fong AH, Leung ST, Chin TW, Lo CSY, et al. Frequency and Distribution of Chest Radiographic Findings in Patients Positive for COVID-19. Radiology. 2020; 296: E72-E78.

[22] Ng MY, Lee EYP, Yang J, Yang F, Li X, Wang H, et al. Imaging Profile of the COVID-19 Infection: Radiologic Findings and Literature Review. Radiology: Cardiothoracic Imaging. 2020; 2: e200034.

[23] Fang Y, Zhang H, Xie J, Lin M, Ying L, Pang P, et al. Sensitivity of Chest CT for COVID-19: Comparison to RT-PCR. Radiology. 2020; 296: E115-E117.

[24] Xie X, Zhong Z, Zhao W, Zheng C, Wang F, Liu J. Chest CT for Typical Coronavirus Disease 2019 (COVID-19) Pneumonia: Relationship to Negative RT-PCR Testing. Radiology. 2020; 296: E41-E45.

[25] Richterman A, Meyerowitz EA, Cevik M. Hospital-Acquired SARSCoV-2 Infection. The Journal of the American Medical Association. 2020; 324: 2155-2156.

[26] Wee LE, Conceicao EP, Sim XYJ, Aung MK, Tan KY, Wong HM, et al. Minimizing intra-hospital transmission of COVID-19: the role of social distancing. Journal of Hospital Infection. 2020; 105: 113-115.

How to cite this article: Sin-Youl Park, Kyung Woo Lee, Suk Hee Lee, Jong Kun Kim, Jae Wan Cho, Jae Cheon Jeon, et al. Prevention of temporary emergency medical center closure through isolation zone and screening triage in COVID-19 outbreak. Signa Vitae. 2022;18(1):34-40. doi:10.22514/sv.2021.111. 